\title{
SISTEMA ESPORTIVO DO REINO UNIDO: DINÂMICA, FUNCIONAMENTO E REFLEXÕES PARA O BRASIL
}

\author{
Fernando Augusto Starepravo \\ Doutor \\ Universidade Estadual de Maringá \\ fernando.starepravo@hotmail.com \\ iD Joe Piggin \\ $\mathrm{PhD}$ \\ Loghborough University \\ J.J.Piggin@lboro.ac.uk
}

Objetivo do estudo: Apresentar o sistema esportivo do Reino Unido, buscando explicar sua dinâmica e funcionamento.

Metodologia: Este estudo utiliza a metodologia proposta por Scheerder, Willem e Claes (2017) e suas cinco questões chave para a compreensão da relação entre Estado e federações esportivas. Os dados foram coletados a partir de banco de dados oficiais, leis, estudos anteriores sobre sistemas esportivos, estatutos de federações esportivas e conselhos e websites de órgãos governamentais e federações esportivas, e analisados com base na análise de conteúdo.

Originalidade/Relevância: Considera-se que existe uma considerável produção de conhecimento sobre políticas de esporte no Reino Unido (UK), mas que a mesma não enfatiza a relação Estado e federações esportivas, e que conhecer mais a fundo esta relação naquele país pode ser profícua a reflexões sobre o sistema esportivo brasileiro.

Principais resultados: O sistema esportivo do Reino Unido baseia-se nos princípios de independência, parcerias e colaboração entre atores de todos os níveis. Todavia, há centralidade de poder em instituições intermediárias, como a UK Sport e a Sport England. Ao passo que o Sistema esportivo do Reino Unido mostra certo equilíbrio no tratamento do esporte de elite e do esporte de massa, no Brasil a ênfase recai sobre o esporte de elite.

Contribuições teóricas/metodológicas: $\mathrm{O}$ trabalho apresenta o sistema esportivo do Reino Unido e inicia a discussão deste em relação ao sistema esportivo brasileiro, tendo como pano de fundo o contexto da globalização.

Palavras-chave: Sistemas esportivos. Federações esportivas. Brasil. Reino Unido.

\section{$\underline{\text { Cite como }}$}

American Psychological Association (APA)

Starepravo, F. A., \& Piggin, J. (2021, jan./abr.). Sistema esportivo do Reino Unido: dinâmica, funcionamento e reflexões para o Brasil. PODIUM Sport, Leisure and Tourism Review, São Paulo, 10(1), 29-55.

https://doi.org/10.5585/podium.v10i1.16400. 


\section{UNITED KINGDOM'S SPORT SYSTEM: DYNAMICS, FUNCTIONING AND REFLECTIONS FOR BRAZIL}

\section{Abstract}

Objective of the study: To introduce the UK sports system, seeking to explain its dynamics and functioning.

Methodology: This study uses the methodology proposed by Scheerder, Willem and Claes (2017) and their five key questions for understanding the relationship between the State and sports federations. We collected data from official databases, laws, previous studies on sports systems, sports federation statutes, and boards and websites of government agencies and sports federations. Then, we analysed it based on the content analysis.

Originality / Relevance: It is possible to acknowledge that there is a considerable production of knowledge about sports policies in the United Kingdom (UK), but that does not emphasize the relationship between the State and sports federations, and that knowing more about that relationship in that country can be useful for reflections on the Brazilian sports system.

Main results: The UK sports system is based on the principles of independence, partnership and collaboration between actors at all levels. However, there is centrality of power in intermediary institutions such as UK Sport and Sport England. While the UK Sporting System shows a certain balance in the treatment of elite sport and mass sport, in Brazil the emphasis is on the elite sport.

Theoretical / methodological contributions: The work presents the UK sports system and starts its discussion in relation to the Brazilian sports system, having the background of globalization.

Keywords: Sports systems. Sports federations. Brazil. United Kingdom.

\section{SISTEMA DEPORTIVO DEL REINO UNIDO: DINÁMICA, FUNCIONAMIENTO Y REFLEXIONES PARA BRASIL}

\section{Resumen}

Objetivo del estudio: Presentar el sistema deportivo del Reino Unido, buscando explicar su dinámica y funcionamiento.

Metodología: Este estudio utiliza la metodología propuesta por Scheerder, Willem y Claes (2017) y sus cinco preguntas clave para comprender la relación entre agencias gubernamentales y federaciones deportivas. Los datos se recopilaron de bases de datos oficiales, leyes, estudios previos sobre sistemas deportivos, estatutos de federaciones y comités deportivos y sitios web de agencias gubernamentales y federaciones deportivas, y se analizaron en función del análisis de contenido.

Originalidad / Relevancia: Considerando que existe una considerable producción de conocimiento sobre políticas deportivas en el Reino Unido (UK), pero que no enfatiza la 
relación entre el Estado y las federaciones deportivas, y que conocer más sobre esa relación en ese país puede ser útil para reflexiones sobre el sistema deportivo brasileño.

Resultados principales: El sistema deportivo del Reino Unido se basa en los principios de independencia, asociación y colaboración entre los actores en todos los niveles. Sin embargo, existe una centralidad de poder en instituciones intermediarias como UK Sport y Sport England. Mientras que el Sistema Deportivo del Reino Unido muestra un cierto equilibrio en el tratamiento del deporte de élite y el deporte de masas, en Brasil el énfasis está en el deporte de élite.

Contribuciones teóricas / metodológicas: El trabajo presenta el sistema deportivo del Reino Unido y comienza su discusión en relación con el sistema deportivo brasileño, en el contexto de la globalización.

Palabras clave: Sistemas desportivos. Federaciones deportivas. Brasil. Reino Unido.

\section{Introdução}

Este estudo versa sobre o sistema esportivo do Reino Unido (UK) com algumas reflexões sobre o sistema esportivo brasileiro. O sistema esportivo do UK foi amplamente discutido por autores como Keech e Nauright (2016), King (2009), Coghlan e Webb (1990), Jefferys (2012), Tacon e Hanson (2011), Houlihan (2000) e Green (2007). Isso porque, segundo Ebbinghaus (2005), devido ao complexo processo de formação dos estados-nação e cooperações internacionais, tipos particulares de países são sobrerrepresentados e outros subrepresentados em análises comparativas. Todavia, há uma lacuna na discussão da relação entre entidades governamentais e federações esportivas (Groeneveld, 2009), exceção feita ao livro de Scheerder, Willem e Claes (2017). Nele, Girginov (2017) apresenta uma discussão sobre o Sistema Esportivo do Reino Unido com ênfase na relação entre governo e federações esportivas.

Já no Brasil, a relação entre Estado e esporte foi estudada, entre outros, por Couto, Couto, Boschi e Lemos (2013), Mazzei, Meira, Bastos, Böhme e De Bosscher (2015), Mezzadri, Silva, Figuêroa e Starepravo (2015) e Starepravo, Canan e Santos (2017), porém nenhum deles abordou centralmente a discussão entre governo e federações esportivas.

Dowling, Brown, Legg \& Grix (2018) apontam que comparações fazem parte da essência do ser humano e, nesse sentido, não é surpresa que estudos comparativos sejam um dos principais interesses para os sociólogos. No campo do esporte são várias as iniciativas de comparação entre sistemas esportivos de países distintos (Scheerder, Willem \& Claes, 2017; Hallmann \& Petry, 2013; Houlihan \& Green, 2008; Nicholson, Hoye \& Houlihan, 2011; Green, 2007; Bergsgard, Houlihan, Mangset, Nødland \& Rommetvedt, 2007; Henry, 2009), com 
especial atenção ao esporte de elite (Andersen \& Ronglan, 2012; Bergsgard, Houlihan, Mangset, Nødland \& Rommetvedt, 2007; Green \& Houlihan, 2005; De Bosscher, Shibli, Westerbeek \& Van Bottenburg, 2015; Houlihan \& Green, 2008; De Bosscher, 2016, entre outros), porém com pouca atenção ao Brasil. Green (2007), por exemplo, desenvolveu uma análise sobre as prioridades das políticas de esporte a longo prazo na Austrália, no Canadá e no Reino Unido. Já De Bosscher (2016) discutiu a utilização de um método de pesquisa misto para estudos comparativos em diferentes países, incluindo o Brasil, no esporte de elite. Bergsgard, Houlihan, Mangset, Nødland e Rommetvedt (2007) identificaram as características da política esportiva em quatro países para determinar se, e em que medida, a política esportiva reflete as características do regime social mais amplo e é afetada por atributos-chave do sistema político doméstico e influências transnacionais, como globalização e comercialização. Henry (2009) identificou e avaliou a natureza e a estrutura dos sistemas de políticas e as mudanças que esses sistemas podem estar sofrendo na União Europeia.

Considerando que existe uma considerável produção de conhecimento sobre políticas de esporte no Reino Unido (UK), mas que a mesma não enfatiza a relação Estado e federações esportivas, e que conhecer mais a fundo esta relação naquele país pode ser profícuo a reflexões sobre o sistema esportivo brasileiro, o objetivo do estudo é apresentar o sistema esportivo do Reino Unido, buscando explicar sua dinâmica e funcionamento. Mais especificamente, nos debruçamos sobre a relação entre o Estado e as federações esportivas, entendendo que tais entidades cumprem papel fundamental no desenvolvimento do esporte em ambos os países. Ao final do texto, são estabelecidas algumas reflexões para pensar o sistema esportivo brasileiro a partir da discussão do sistema britânico, apontando uma nova agenda de pesquisa.

\section{Antecedentes históricos da política de esporte no Reino Unido}

A relação entre esporte e política emergiu, segundo Keech e Nauright (2016) e King (2009), durante o período pós Segunda Guerra Mundial. Antes disso, o esporte no UK era considerado uma atividade voluntária, parte da longa tradição do voluntarismo na sociedade em geral. Durante os anos 1950, as National Governing Bodies (NGBs), algo próximo às nossas confederações, passaram a solicitar mais recursos públicos para dar conta da demanda crescente por esporte. Também crescia, naquele momento, o apoio daqueles que viam o esporte como veículo de coesão e integração social. Governos sucessivos passaram a considerar o esporte um 
mecanismo cada vez mais importante de política social, através do qual se poderia lidar com questões relacionadas especialmente aos jovens (Keech \& Nauright, 2016). Já para Coghlan e Webb (1990), até a década de 1960, o governo central britânico se envolveu pouco ou não tomou parte no esporte. Posição corroborada por Jefferys (2012), para quem, entre 1945 (ano do final da Segunda Guerra Mundial) e 1964, políticas de esporte não existiram.

Houlihan (1991) fala do movimento em torno da criação de um conselho de desenvolvimento esportivo (Sports Council), sugerido pela primeira vez no relatório Wolfenden Sport in the Community (1960), que poderia financiar e supervisionar o desenvolvimento do esporte para todos e do esporte de elite. Por outro lado, Jefferys (2012) fala que até a saída do Partido Conservador do poder, em 1964, nada de concreto havia sido realizado para a criação do Conselho. Para o autor, Harold Wilson, trabalhista, foi o primeiro Primeiro-Ministro a perceber o potencial eleitoral do esporte. Em 1964, o Governo nomeou o primeiro Ministro com responsabilidades para com o Esporte, Lord Hailsham, cuja principal responsabilidade era supervisionar o desenvolvimento do conselho consultivo de desenvolvimento esportivo, estabelecido em 1965 (Keech \& Nauright, 2016), sob um governo trabalhista, como órgão consultivo, e transformado, em 1972, sob um governo conservador, em um Conselho com funções executivas (Tacon \& Hanson, 2011; Keech \& Nauright, 2016).

Segundo Jefferys (2012), as administrações trabalhistas e conservadoras entre os anos de 1964 e 1979 continuaram a impulsionar o desenvolvimento do esporte. Para Houlihan (1991), as políticas esportivas nesse período emergiram pautadas em questões como o sucesso esportivo internacional e problemas sociais, como transtornos da juventude e pressões eleitorais para a expansão de instalações esportivas financiadas pelo Estado. O foco no esporte de participação esteve relacionado, segundo King (2009), às diretrizes do Conselho Europeu sobre o Sport for All.

Mesmo que, segundo Houlihan (1991), houvesse uma tensão entre os interesses do esporte de elite e do esporte para todos, houve um consenso que o investimento em instalações esportivas seria a primeira prioridade. Segundo King (2009), entre 1972 e 1978, o número de centros municipais de esporte se multiplicou e o número de piscinas cresceu mais de $70 \%$. Segundo Jefferys (2012), entre 1973 e 1977, as instalações para esportes indoors quase triplicaram, havendo especial incremento no número de Centros de Lazer, de 12 em 1971 para 449 em 1981. De acordo com Houlihan (1997), o Sports Council destinou, entre 1972 e 1976, cerca de 4,7 milhões de libras para uma variedade de instalações esportivas e de lazer. As novas instalações, financiadas pelo Sports Council, garantiram uma massiva expansão do esporte. 
Centros locais de esporte e lazer quadruplicaram durante a década de 1970, chegando a mais de mil no final da década (Tacon \& Hanson, 2011).

Em suma, segundo King (2009), as autoridades locais foram colocadas no centro da política, do planejamento e da provisão de esportes locais, e, apesar das mudanças nos governos locais desde a década de 1970, continuam sendo os maiores contribuidores financeiros para o esporte e a recreação.

A década de 1980 foi marcada pela indiferença governamental em relação ao esporte, especialmente pela Primeira-Ministra Margaret Thatcher e sua agenda neoliberal (Jefferys, 2012). Para o autor, o esporte de elite não podia confiar no apoio do governo às candidaturas de Birmingham e Manchester em receber os Jogos Olímpicos, enquanto internamente o Sports Council tinha dificuldade em manter as ações de anos atrás, havendo críticas de setores conservadores quanto à necessidade de um Conselho ou Ministério do Esporte. De acordo com Houlihan (2000), para a Primeira-Ministra, a participação em massa no esporte era um serviço que cada usuário deveria pagar, enquanto o esporte de elite, especialmente o futebol, era mais uma fonte de problemas do que uma fonte de orgulho. Já King (2009) destaca que o período Thatcher foi marcado pela gradual redução dos recursos disponíveis para autoridades locais e conselhos de esporte.

Nos anos 1990, segundo Tacon and Hanson (2011), o status do esporte no governo melhorou. O British Sports Council foi substituído por um UK Sports Council e um English Sports Council - agora conhecidos como UK Sport e Sport England. Ainda segundo os autores, a criação da Loteria Nacional em 1994 foi crucial para a gestão esportiva no Reino Unido, levando à criação de instalações de ponta nas autoridades locais e à atualização de instalações em muitos clubes esportivos. Também nessa década houve a introdução de financiamento para atletas de elite, precipitando um processo de modernização em muitos órgãos de governo, que até então não haviam recebido quantias significativas de dinheiro público e agora precisavam se adaptar às suas novas responsabilidades.

De acordo com Green (2004), em 1995, o governo conservador publicou uma declaração política abrangente: Sport: Raising the Game (UK, 1995), que indicava a retirada do governo central e dos conselhos esportivos da provisão de oportunidades para participação em massa e focada, entre outras coisas no (i) desenvolvimento de atletas de elite e um instituto esportivo de elite; (ii) estabelecimento do papel das instituições de ensino superior na promoção de atletas de elite; e (iii) na alocação de recursos a entidades esportivas, que agora dependem do apoio explícito aos objetivos do governo (Houlihan, 1997). Segundo Green (2004, p. 371), 
como Lentell argumentou no início dos anos 1990, "Parece que o Conselho está encerrando sua 'ligação perigosa' com o mundo da 'Comunidade' para se juntar ao mundo mais confortável do 'Esporte'". Segundo Tacon and Hanson (2011), de 1990 a 2010, houve avanços significativos na política esportiva, foram disponibilizadas novas fontes de receita significativas para o esporte de elite e de massa por meio da introdução da Loteria Nacional.

Em síntese, seguindo Collins (2008), cinco principais fases da política esportiva no Reino Unido podem ser distinguidas: Desenvolvimento da base de instalações e gerenciamento de instalações (1960 a 1982); Direcionamento e desenvolvimento esportivo precoce nacional e regional (1982-1991); Mudança de prioridades para desempenho e excelência (1992-1997); Inclusão social e mais medalhas (1997-2015); e "Nação ativa: aprimorando o valor do esporte para os indivíduos em 2016-?". Porém, para o autor, a lógica ideológica da política esportiva do Reino Unido permaneceu relativamente inalterada e, apesar das variações políticas, gira em torno da preocupação expressa de usar o esporte para moldar o caráter e promover o bem social na forma de melhor saúde, educação, prestígio nacional, economia, desenvolvimento e cidadania.

\section{Fundamentação teórica: desvendando a relação entre Estado e (con)federações esportivas}

Via de regra federações ou confederações esportivas (usaremos a primeira denominação no decorrer do texto) são um tipo de órgão dirigente de esporte (sports governing body em inglês) responsáveis pela representação e organização do esporte em uma determinada porção geográfica. Caracterizam-se em geral como entidades privadas do terceiro setor ${ }^{1}$, uma vez que não têm como finalidade principal a obtenção de lucros a serem distribuídos entre seus associados.

As relações entre governo e entidades do terceiro setor no campo do esporte já foram estudados por diferentes autores e em diferentes perspectivas (Walker \& Hayton, 2018; Parnell, May, Widdop, Cope \& Bailey, 2019; Vos, Wicker, Breuer \& Scheerder, 2013; Thing \& Ottesen, 2010; Giulianotti, Itkonen, Nevala \& Salmikangas, 2019; Skille, 2015). Há uma tendência a

\footnotetext{
${ }^{1}$ Precisamos reconhecer, como Kendall (2010) e Stephenson (2013), que a noção de "terceiro setor" é variada, controversa e geralmente usada para descrever entidades entre o mercado e o estado, incluindo "ONGs" e "sociedade civil organizada". Para Simaens e Koster (2013), as organizações do terceiro setor geralmente compreendem um conjunto de organizações entre o mercado, o estado e a comunidade; incluindo organizações sem fins lucrativos, associações, ONGs, sociedades de benefício mútuo e algumas fundações, entre outras. Salamon e Flaherty $(1996$, p. 4) são mais precisos ao definir "organização sem fins lucrativos" como uma ampla gama de entidades que "atendem a cinco requisitos cruciais: elas são organizadas, não fazem parte da estrutura do estado, não fazem distribuir lucros aos seus membros; eles são autônomos; e envolvem alguma contribuição voluntária significativa".
} 
estudar tais relações em contextos de austeridade econômica (como em Walker \& Hayton, 2018 e Parnell, May, Widdop, Cope \& Bailey, 2019) ou em regiões onde o associativismo e a participação são importantes fatores de coesão social (como em Vos, Wicker, Breuer \& Scheerder, 2013 e Giulianotti, Itkonen, Nevala \& Salmikangas, 2019). Entretanto, as relações entre federações esportivas e agências governamentais são negligenciadas por esse subcampo de pesquisa (Groeneveld 2009), exceto pelo livro de Scheerder, Willem e Claes (2017) e pelo artigo de Groeneveld (2009).

Buscando avançar no entendimento do relacionamento entre governo e federações esportivas, Scheerder, Willem e Claes (2017) distinguem duas principais formas que a relação pode assumir: (i) um relacionamento direto entre o governo e as federações esportivas; e (ii) relacionamentos nos quais instituições atuam como intermediárias da relação entre governo e federações esportivas. Para entender esses diferentes tipos de relação, os autores apresentam duas teorias distintas: abordagem do agente principal e da cogovernança.

Para a abordagem do agente principal, nos relacionamentos, o "agente", atua em nome ou como representante do outro, designado "principal", em um domínio particular de decisões. Para essa abordagem, o relacionamento é inerentemente problemático, pois o principal tem menos acesso às informações. Existe um conflito de interesses e assimetria de informações que permite ao agente servir seus próprios interesses em detrimento do principal. No entanto, é necessário não simplificar as complexas relações políticas envolvidas, inclusive nas políticas esportivas, afinal, Shapiro (2005) aponta que o sistema político deve ser entendido como uma complexa rede de relações principal-agente em que os atores, ao mesmo tempo, podem desempenhar papéis como principais e como agentes.

Segundo Scheerder, Willem e Claes (2017), uma segunda teoria implica a perspectiva de cogovernança, sugerindo interação direta entre o governo e as federações esportivas no desenvolvimento de políticas esportivas, em um relacionamento permanente. Também para Groeneveld (2009), a cogovernança envolve interação direta no setor público e significativa entre o Estado, federações e cidadãos em termos reais nos processos de formulação e implementação de políticas. Devido à pequena quantidade de pesquisas sobre a relação entre federações esportivas e o Estado, essa teoria permanece um tanto conceitual e não prática em nossa área. Somerville e Haines (2008) explicam que a cogovernança pode ocorrer em qualquer escala ou em várias escalas. Além disso, apontam que a cogovernança pode ocorrer em qualquer arena em que um coletivo opere. No entanto, a governança política envolve especificamente três tipos de arenas: arenas governamentais, arenas não-governamentais e arenas de novos tipos, 
nas quais atores governamentais e não-governamentais se encontram para debater, decidir e agir em conjunto, como parece ser o caso do esporte.

Outra teoria importante para entendermos a relação entre governo e federações esportivas é a Teoria da Dependência de Recursos, para a qual a chave da sobrevivência organizacional é a capacidade de adquirir e manter recursos (Pfeffer \& Salancik, 1978). Segundo Davis e Cobb (2009), a teoria pode ser resumida como a escolha do dispositivo menos constrangedor para governar as relações com seus parceiros de troca, o que permitirá maximizar sua autonomia e minimizar a incerteza e a dependência. Enquanto estudava o esporte de elite, Lucidarme, Babiak e Willem (2018) sugeriram que o sucesso esportivo internacional só é possível quando existe uma colaboração eficaz entre os membros de uma rede. As organizações precisam negociar com os parceiros para reduzir a incerteza e permitir a troca de recursos.

Nesse sentido, os autores usaram a Teoria da Dependência de Recursos para explicar como interdependências e desequilíbrios de poder surgem nas redes esportivas de elite, estudando a complexa gama de relações múltiplas e interdependentes existentes. Quando cada organização não consegue gerar internamente todos os recursos necessários, é preciso negociar com outras organizações para obter acesso a recursos significativos. Portanto, a autonomia organizacional é diminuída, a capacidade de operar de forma independente é reduzida e a independência na tomada de decisões é restrita (Lucidarme, Babiak \& Willem, 2018).

\section{Metodologia}

Este estudo utiliza a metodologia proposta por Scheerder, Willem e Claes (2017) e suas cinco questões chave para a compreensão da relação entre Estado e federações esportivas. Primeiro, um breve panorama do país é providenciado, descrevendo as características geográficas, demográficas, políticas, econômicas e socioculturais, bem como o nível de participação esportiva da população e outras características do esporte no país. Segundo, são analisadas as estruturas do Sistema esportivo com base na estrutura utilizada por Hallmann e Petry (2013), incluindo estruturas governamentais, intermediárias e não-governamentais a nível nacional, regional e local. Depois, são discutidas como as federações esportivas são dirigidas por órgãos governamentais e como as federações suportam suas operações. Cada estudo é finalizado, então, apontando similaridades e diferenças em relação a outros países, conforme imagem abaixo: 
Figura 1 - Metodologia para análise da relação entre federações esportivas e órgãos governamentais

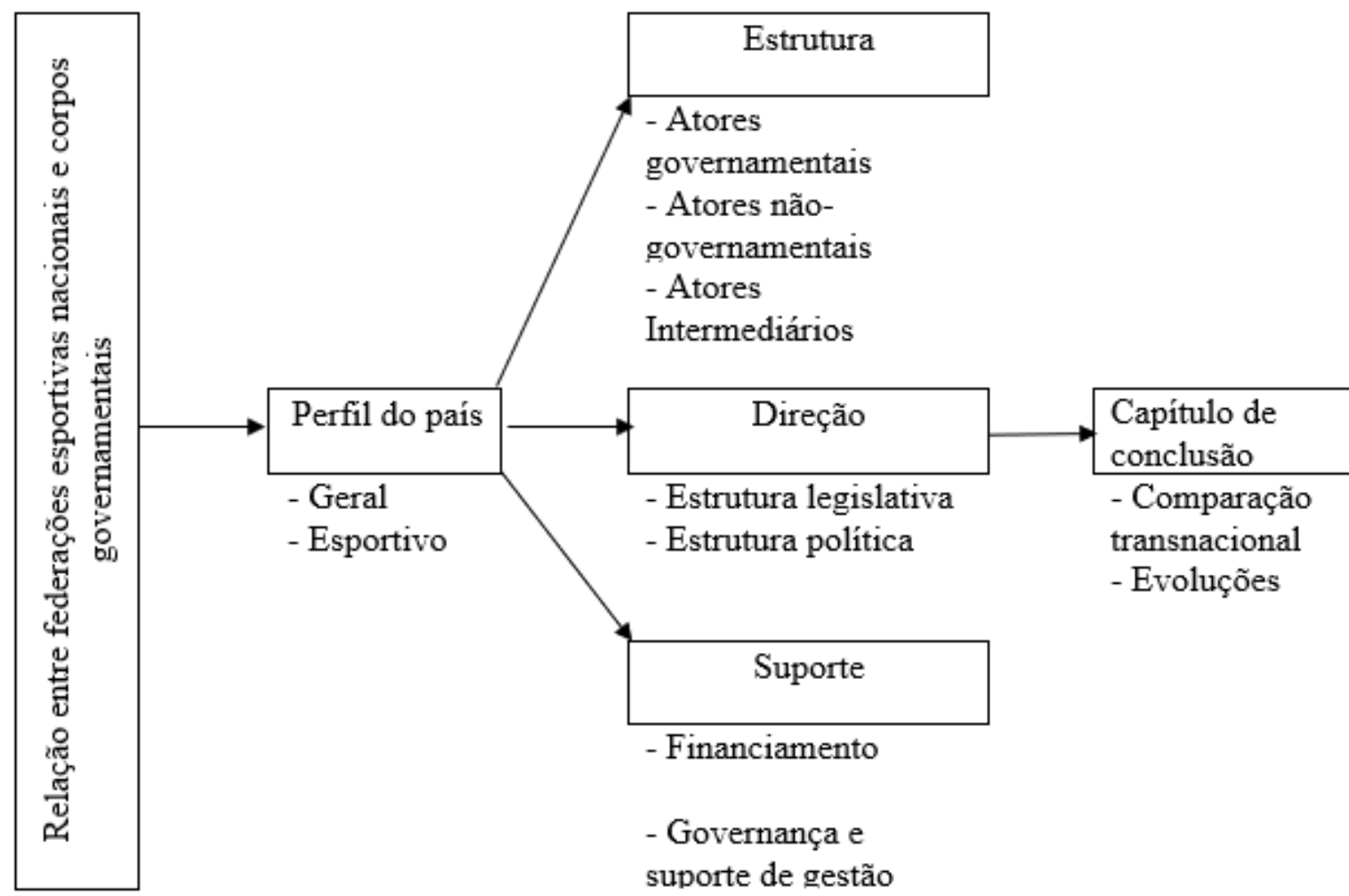

Fonte: Adaptado de Scheerder, Willem e Claes (2017).

Considerando as reflexões de Dowling, Brown, Legg e Grix (2018), reconhecemos as limitações metodológicas em análises comparativas de sistemas esportivos, incluindo a de Scheerder, Willem e Claes (2017), mas entendemos que essa metodologia pode ser um guia para o entendimento dos sistemas esportivos em diferentes países, já tendo sido aplicada ao menos em treze diferentes países europeus.

Os dados foram coletados a partir de banco de dados oficiais, leis, estudos anteriores sobre sistemas esportivos, estatutos de federações esportivas e conselhos e websites de órgãos governamentais e federações esportivas.

Todas as informações disponíveis foram consideradas e as pesquisas continuaram até a saturação dos dados. Primeiramente, foram coletados os dados, lidas todas as informações e selecionadas partes específicas que interessavam ao escopo da pesquisa. Posteriormente, os dados foram classificados de acordo com a análise de conteúdo. Devido ao volume de dados coletados, agrupamos os dados em grandes categorias, por vezes exemplificadas por citações pontuais. 
Starepravo, F. A., \& Piggin, J. (2021, jan./abr.). Sistema esportivo do Reino Unido: dinâmica, funcionamento e reflexões para o Brasil

\section{Resultados e discussão: Sistema Esportivo do Reino Unido e reflexões ao contexto brasileiro}

A primeira questão que devemos ter em mente é que, apesar de ser tratado nos estudos sobre sistemas esportivos como uma unidade, o Reino Unido é composto por quatro diferentes nações - Inglaterra, País de Gales, Escócia e Irlanda do Norte -, que apresentam similaridades e diferenças entre si. Girginov (2017) e Gratton, Taylor e Rowe (2013), por exemplo, problematizam essa questão e focam suas análises na Inglaterra. De certa forma, faremos algo semelhante ao focar nossa análise na Inglaterra, principal país do bloco que concentra cerca de $85 \%$ da população do UK, sem, porém, desconsiderar o UK como uma unidade que, no conjunto, afeta as políticas esportivas de seus países membros. Isso porque, segundo UK (2015), o governo do Reino Unido é responsável pelo esporte de elite tanto no nível do UK quanto na Inglaterra, com os governos descentralizados da Escócia, País de Gales e Irlanda do Norte sendo responsáveis pelo esporte de elite em seus próprios países. O governo do UK também é responsável por uma série de questões reservadas, como segurança ou antidoping em todo o Reino Unido, enquanto a política de esporte, saúde e educação de base é desenvolvida na Escócia, no País de Gales e na Irlanda do Norte.

Em 2018, o Reino Unido tinha uma população estimada de 66,4 milhões de pessoas, das quais aproximadamente 56 milhões vivem na Inglaterra (Ons, 2019). Sua população é predominantemente urbana (mais de 80\%) e apresenta altos índices de envelhecimento (a expectativa de vida em 2017 era de 81,1 anos). O Produto Interno Bruto (PIB) per capita, em 2018, foi de US\$ 41.770,00 - no mesmo ano, o Brasil alcançou US\$ 9.140,00 (World Bank, 2019). O país tem enfrentado desafios nas finanças públicas, resultando em um débito de 1,8 trilhão de libras, que corresponde a cerca de 90\% do PIB, em novembro de 2019 (Ons, 2019). Tais desafios têm gerado uma série de cortes significativos nos gastos públicos, os quais impactam fortemente o sistema esportivo.

Segundo dados da Sport England (2019a), 62,6\% dos ingleses adultos são considerados ativos (praticam algum tipo de atividade física por mais de 150 minutos por semana), enquanto 25,1\% são considerados inativos (menos de 30 minutos de atividade física por semana). A participação é maior entre os homens (65\%) em relação às mulheres (61\%). Os índices de pessoas inativas aumentam de acordo com a idade, partindo de $15 \%$ entre jovens de 16 a 24 anos, chegando a $70 \%$ entre idosos com mais de 85 anos. A inatividade entre as pessoas com deficiência é o dobro em comparação com as pessoas sem deficiência (41\% x 21\%). As 
atividades físicas mais praticadas são a caminhada, atividades fitness, corrida, atletismo e esportes múltiplos (Sport England, 2019a).

O UK conta com aproximadamente 151.000 clubes, mais de três milhões de adultos que se voluntariam no esporte por pelo menos uma hora por semana e uma associação ativa de mais de 5 milhões de pessoas. O setor esportivo emprega aproximadamente 400.000 pessoas na Inglaterra, atrai cerca de 1,7 bilhão de euros em gastos públicos a cada ano e atende a mais de 9,1 milhões de pessoas membros de um clube esportivo, número que representa $21 \%$ da população inglesa (Girginov, 2017).

O segundo aspecto da análise, seguindo o modelo de Scheerder, Willem e Claes (2017), é a estrutura do esporte organizado com base no modelo utilizado por Hallmann e Petry (2013), incluindo estruturas governamentais (governamental), intermediárias (intermediate) e não governamentais (non-governmental) nos níveis nacional (national level), regional (regional level) e local. Segundo Girginov (2017) e Gratton, Taylor e Rowe (2013), o sistema esportivo do Reino Unido seria organizado da seguinte forma:

Figura 2 - Organização do esporte no Reino Unido

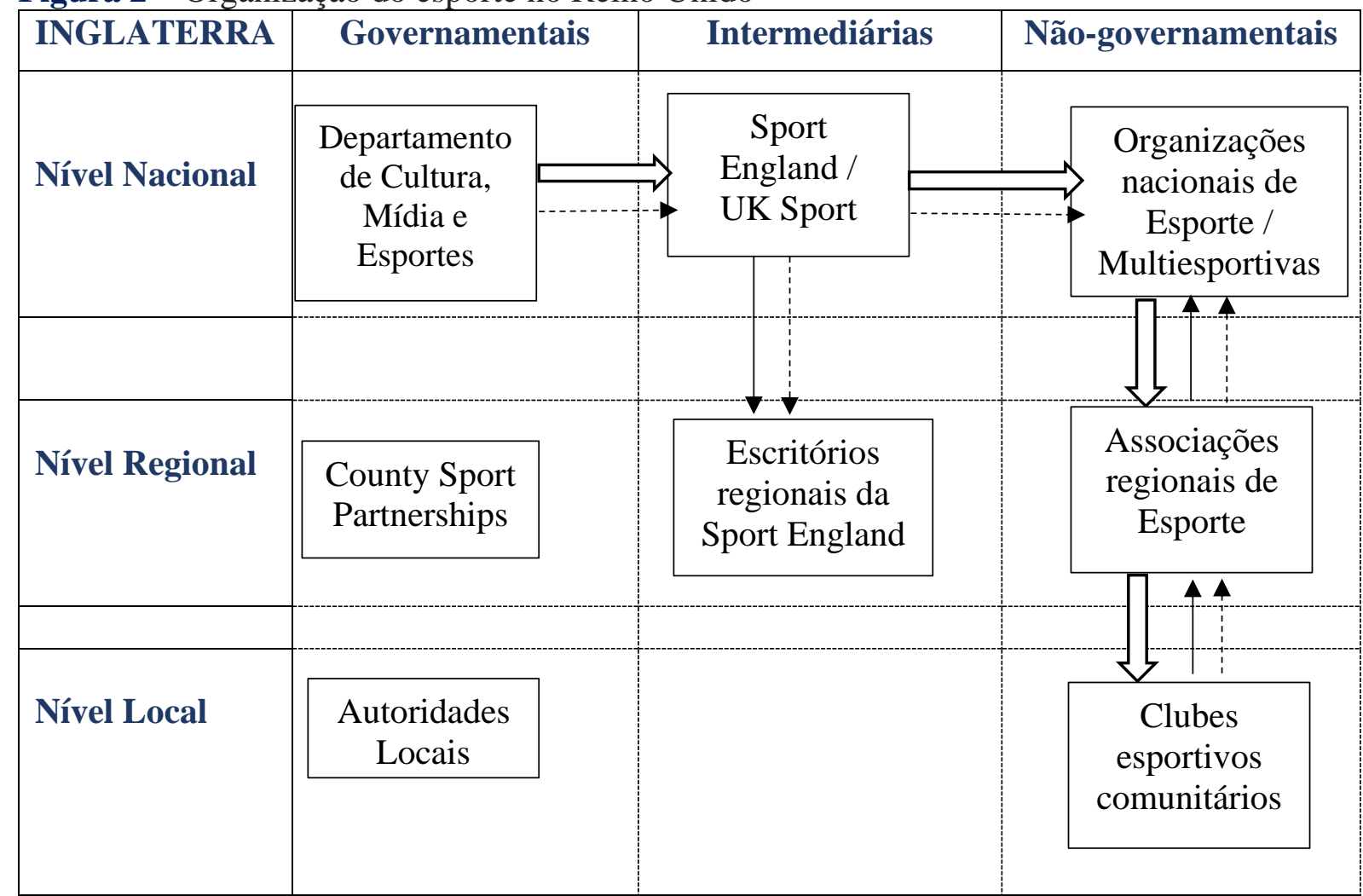

Financiamento

Filiação / Parceria

Relação hierárquica

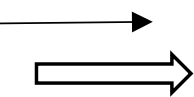

Fonte: Adaptado de Girginov (2017) e Gratton, Taylor e Rowe (2013). 
Starepravo, F. A., \& Piggin, J. (2021, jan./abr.). Sistema esportivo do Reino Unido: dinâmica, funcionamento e reflexões para o Brasil

O Departamento de Cultura, Mídia e Esportes (DCMS) é o principal órgão governamental responsável pelo esporte no UK e na Inglaterra, em particular. A política do DCMS prevê o auxílio à política de esporte para todos ao mesmo tempo em que ambiciona o sucesso do esporte de elite.

O sistema esportivo inglês, segundo Girginov (2017), baseia-se nos princípios de independência, parcerias e colaboração entre atores de todos os níveis. A independência da Sport England e da UK Sport é garantida por seu status quase não-governamental, conforme estabelecido por uma Carta Real, a qual, em teoria, fornece um certo grau de independência do governo. Entretanto, ambas as organizações são quase completamente financiadas pelo governo e prestam contas a ele.

A Sport England tem como principal função ajudar as pessoas e comunidades em todo o país a adquirirem um hábito esportivo para toda a vida (Sport England, 2019c), enquanto a UK Sport é a agência para o esporte de elite do país que investe no esporte olímpico e paralímpico. Sua missão é trabalhar em parceria para levar o esporte no UK ao sucesso esportivo em nível mundial (UK Sports, 2019). A UK Sport é responsável por gerenciar e distribuir o investimento público, e é distribuidora estatutária de fundos arrecadados pela Loteria Nacional para o esporte de elite. Em contrapartia, a Sport England investe as receitas permitidas da Loteria Nacional e do Tesouro em organizações e projetos que visam aumentar a participação no esporte de base e criar oportunidades para as pessoas se destacarem no esporte escolhido (Girginov, 2017).

O papel do governo central é definir a política de alto nível que orienta como o dinheiro público é investido, ao invés de tomar todas as decisões de financiamento. Segundo UK (2015), essa distância é particularmente importante no esporte, onde são necessários conhecimentos especializados para tomar as melhores e mais embasadas decisões, guiadas pelas necessidades do público-alvo. Nesse sentido, o governo geralmente não prescreve quais organizações, esportes ou tipos de atividades devem ser financiados; esse é o papel da UK Sport, Sport England, Public Health England (PHE), entre outros (UK, 2015).

As principais instituições guarda-chuva, chamadas de National Sport / Multisport Organization por Girginov (2017) e Gratton, Taylor e Rowe (2013), são as National Governing Bodies ou National Federations for individual sports (NGBs), a Sport and Recreation Alliance (SRA), a British Olympic Association (BOA) e a British Paralympic Association (BPA). 
As NGBs são tipicamente organizações privadas, independentes e autonomeadas, que governam seus esportes através do consentimento da comunidade envolvida naquela modalidade, algo semelhante às confederações esportivas no Brasil. Há um processo de reconhecimento das NGBs, o qual envolve a UK Sport e os quatro Conselhos Esportivos dos diferentes países que compõem o Reino Unido, com o objetivo de identificar uma única estrutura líder de NGB que governe determinado esporte no Reino Unido ou no país de origem (Sport England, 2019b).

Segundo Houlihan e White (2002), muitas das NGBs foram estabelecidas como organizações voluntárias no século XIX, com a responsabilidade de regular seus esportes e as competições esportivas. Mais recentemente, as responsabilidades das NGBs se expandiram, incluindo o envolvimento com instalações esportivas, eventos e uma ênfase maior no desenvolvimento do esporte como um todo, desde a base até a elite (Houlihan \& White, 2002). Para Walters, Tacon e Trenberth (2011), o papel atualizado das NGBs envolve: organização e gerenciamento de competições, desenvolvimento de treinadores, aumento da participação esportiva, desenvolvimento de talentos, treinamento de voluntários, marketing e promoção do esporte, entre outras.

As Associações Olímpicas e Paralímpicas Britânicas são responsáveis por promover o movimento olímpico e paralímpico no Reino Unido. São instituições independentes do governo, não recebem financiamento do Estado ou da Loteria Nacional e são financiados com seus próprios fundos mobilizados de forma privada, comercial ou através da participação do público (BOA, s.d.; ParalympicsGB, s.d.).

Segundo Girginov (2017), a Sport and Recreation Alliance é uma organização guardachuva independente que inclui o governo e a representação de 320 órgãos de esporte e recreação. Seu objetivo é garantir que o esporte e a recreação permaneçam no topo da agenda política no Reino Unido. Representa mais de 150.000 clubes e 8 milhões de participantes regulares de esportes ou associados de clubes no nível do Reino Unido.

No nível regional, as County Sports Partnerships (43 na Inglaterra) (Active Partnership, s. d.), segundo Gratton, Taylor e Rowe (2013), financiadas pela Sport England e por outras parcerias, foram criadas para fornecer coordenação estratégica em suas áreas geográficas. Elas o fazem buscando criar um entendimento profundo das necessidades da comunidade local, de forma a intermediar parcerias intersetoriais, apoiar o desenvolvimento de força de trabalho, engajar comunidades e compartilhar a aprendizagem do que funciona localmente, com especial 
atenção a pessoas inativas e a grupos sub-representados que se beneficiarão ainda mais de um estilo de vida ativo (Active Partnership, s. d.).

Elas desempenham, segundo o governo britânico, um papel importante em todo o país na promoção do esporte e da atividade física, trabalhando em estreita colaboração com as autoridades locais, escolas e outros. Ainda segundo o governo, à medida que o governo local evolui, faz-se necessário pensar nas melhores maneiras de fazer com que as organizações locais trabalhem juntas para fornecer as prioridades em suas áreas, de maneira que as pessoas possam tirar o máximo proveito do esporte (UK, 2015).

Os Escritórios regionais da Sport England são 12 estruturas descentralizadas da Sport England em diferentes regiões da Inglaterra. Elas são tratadas como iniciativas-piloto que têm a finalidade de entender as barreiras que as pessoas enfrentam para serem ativas e como se pode desenvolver soluções sustentáveis regionalmente (Sport England, 2019c).

As autoridades locais na Inglaterra, segundo Girginov (2017), não têm o dever estatutário de fornecer serviços esportivos e recreativos, mas até o final dos anos 1990 elas eram consideradas o principal fornecedor de comunidades locais, investindo coletivamente até 1,5 bilhão de libras por ano em esportes. Dados mais atuais apontam que as autoridades locais são os maiores investidores do setor público em esportes e atividades físicas, gastando mais de 1 bilhão de libras por ano, excluindo os gastos de capital (UK, 2015). Eles trabalham em estreita colaboração com NGBs e Sport England, administram uma variedade de instalações esportivas locais e fornecem apoio ao esporte em todo o país.

Essa atuação das autoridades locais, segundo Girginov (2017), estaria relacionada às crenças nos efeitos positivos do esporte na coesão da comunidade, redução do crime juvenil, benefícios à saúde e regeneração econômica. Tal tipo de posicionamento pode ser verificado nas palavras do então Ministro dos Governos Locais, Marcus Jones:

O esporte pode ser a cola que mantém as comunidades unidas e é uma ferramenta persuasiva na promoção de interesses compartilhados, além de promover um forte senso de orgulho cívico. Eu já vi em primeira mão o poder positivo que o esporte e a recreação exercem, de crianças de todas as origens imitando seus heróis nas instalações da comunidade local a apoiadores trabalhando juntos para proteger os bens que valorizam (UK, 2015, p. 13, tradução nossa).

Segundo Gratton, Taylor e Rowe (2013), as organizações locais estão no centro do fornecimento de esportes na Inglaterra, sendo os clubes esportivos e as autoridades locais os mais significativos. Os autores mencionam que existiam, em 2006, 3.489 instalações esportivas 
e de lazer com acesso público na Inglaterra, das quais três quartos pertenciam às autoridades locais.

Segundo UK (2015), o esporte é uma parte essencial das comunidades locais, mas se apresenta de maneira diferente em cada lugar, não existindo uma abordagem de cima para baixo que funcione em todos os lugares. Enquanto alguns resultados podem ser encomendados nacionalmente, locais diferentes exigem estratégias próprias que respondam à necessidade local e definam parcerias localmente, uma vez que a compreensão do poder local acerca das comunidades lhes permite visar oportunidades e incentivar a participação em massa (UK, 2015).

Ainda no nível local, segundo Girginov (2017), existem, em geral, dois tipos de clubes esportivos na Inglaterra: os clubes esportivos comunitários e os esportivos não-comunitários. Os primeiros são frequentemente baseados em centros comunitários e podem ser registrados como independentes. Já os últimos são claramente afiliados a uma federação esportiva. Como mencionado, existem na Inglaterra cerca de 151.000 clubes esportivos individuais. Com isso, o cenário esportivo na Inglaterra é formado por muitos clubes pequenos, sem poder real e com recursos limitados. No entanto, uma vez que um clube se torna parte do Community Amateur Sports Club, ele se beneficia de um tratamento tributário mais favorável (Girginov, 2017).

Em relação à terceira questão-chave de Scheerder, Willem e Claes (2017), acerca de como as federações esportivas são dirigidas por corpos governamentais, no que diz respeito à legislação, o governo do Reino Unido adotou uma abordagem não intervencionista do esporte e não existe uma lei geral do esporte. Em vez disso, a regulamentação dos esportes individuais é largamente deixada aos seus órgãos governamentais nacionais, as NGBs (Lexology, 2019).

Para Girginov (2017), a maior parte da legislação relativa ao esporte é permissiva no sentido de aceitar (mas não exigir) que diferentes níveis de governo sejam ativos no campo e no financiamento do esporte. Dessa maneira, muitos atores estão envolvidos ativa e livremente. Cabe lembrar que o sistema esportivo inglês se baseia nos princípios de independência, parcerias e colaboração entre atores de todos os níveis.

Segundo Henry (2009, p. 45), a estrutura de política esportiva na Inglaterra é caracterizada como empreendedora, a qual "se concentra quase exclusivamente em resultados, particularmente no contexto de órgãos do setor público que contratam entidades comerciais para gerenciar serviços, onde os contratos estipularão os tipos de resultados a serem alcançados". Segundo Girginov (2017), a filosofia subjacente a este sistema tem sido os princípios da Nova Gestão Pública, com suas preocupações expressas com eficiência e metas. 
Ainda segundo o autor, embora todas as NGBs no Reino Unido sejam bastante independentes do Estado, eles continuam a receber entre $60 \%$ e $70 \%$ de seu financiamento do governo, principalmente através da Sport England, colocando-as em um relacionamento de dependência de poder.

O financiamento da maioria das organizações esportivas britânicas, quarta questão chave de Scheerder, Willem e Claes (2017), segundo Girginov (2017), depende em grande parte das contribuições do governo, seja diretamente (por meio de fundos do Tesouro) ou indiretamente (por meio da renda da Loteria Nacional, licenciada pelo governo do Reino Unido).

O financiamento das NGBs, segundo Girginov (2017), varia muito, dependendo da viabilidade comercial do esporte. Para clubes e federações, as taxas de associação são importantes. A maioria das federações também vende mercadorias específicas. Eles também ganham dinheiro com a realização de diferentes eventos. No entanto, o financiamento do esporte de base é uma responsabilidade central do Sport England. Esse suporte financeiro é baseado nos Whole Sport Plans, desenvolvido por cada NGB em relação a três áreas distintas: (1) aumento da participação de jovens de 14 a 25 anos; (2) aumento da participação de adultos acima de 25 anos; e (3) talento esportivo. Tanto a Sport England quanto a UK Sport financiam apenas as NGBs que atuam com esportes olímpicos ou paralímpicos, seguindo orientações da política estratégica do governo do Reino Unido.

Partindo para a análise dos dados e considerando o referencial teórico anunciado, podemos, num primeiro momento, analisar o Sistema Esportivo do Reino Unido no que diz respeito às relações entre o governo e as federações esportivas como uma relação mediada, não direta. O governo se relaciona diretamente e mais fortemente com duas instituições, a UK Sport e a Sport England. Tais instituições recebem recursos públicos diretos e indiretos, oriundos da Loteria Nacional e do Tesouro, e repassam às NGBs. Atuam, portanto, como "agentes", em nome ou representando as NGBs, os "principais".

Recortando o olhar apenas para a relação entre os órgãos governamentais e os intermediários, podemos avaliar que essa é uma relação prática e objetiva, envolvendo um pequeno número de agentes. Para o governo, parece muito mais simples se relacionar com um número limitado de instituições, as quais receberão o recurso financeiro e deverão prestar contas sobre ele. Por outro lado, o poder de decisão de como esses recursos serão distribuídos e o que será priorizado em termos de desenvolvimento esportivo fica nas mãos dessas instituições "agentes". 
Ao visitar os documentos oficiais e a literatura, podemos dizer que essa questão de repasse das decisões a um órgão não-governamental não é um problema, pois reconhece-se naquele país a autonomia e a diversidade da organização esportiva, calcada em uma cultura associativista, a qual poderia dar conta de direcionar os investimentos dos recursos públicos em esporte. Houlihan (1991, pp. 83, tradução nossa), por exemplo, afirma que o governo usou alguns mecanismos para manter certo controle, por meio dos quais não tivesse que se envolver demais: "o esporte, como as artes, não era visto como uma esfera apropriada para a ação política ou direta do governo e, consequentemente, o envolvimento do governo tem sido mediado por meio de órgãos quase governamentais", como a UK Sport e a Sport England.

Além disso, o governo, por meio de planos e estratégias, como o Sporting Future: A New Strategy for an Active Nation (UK, 2015), deixa claro as diretrizes e metas a serem alcançadas. Com isso, o Estado parece fazer valer seus interesses com o esporte, como identidade nacional, instrumento de educação dos jovens e apoio ao voluntariado, sem, contudo, centralizar a tomada de decisão, relacionando-se com um número limitado de players. Segundo Collins (2010), embora as NGBs sejam formalmente independentes do governo, políticas e financiamento público - incluindo planos de responsabilizar as NGBs pela produção de resultados, focados não só no sucesso das medalhas, mas também na participação crescente em troca de dinheiro público - fizeram com que elas se tornassem menos independentes.

Ao olhar para a relação entre as estruturas intermediárias, UK Sport e Sport England, e as federações, observamos que as primeiras, no papel de "agentes", detêm o acesso privilegiado a informações, o que gera um cenário de conflito de interesses e assimetria de informações, o qual permite ao agente servir seus próprios interesses em detrimento do principal. Isso pode ser verificado na insatisfação de parte das NGBs em relação à $U K$ Sport.

Reportagem publicada pela BBC, em 2018, apresenta queixas à UK Sport, que busca controlar as NGBs através do uso de ameaças em "flagrante desrespeito" ao seu código de governança esportiva, agindo além de seus poderes. Rowland-Jones - um ex-coronel do Exército - fez alegações semelhantes em sua carta, alegando que "um número crescente de NGBs está insatisfeito com a maneira como a UK Sport está se comportando" (Roan \& Nathanson, 2018, tradução nossa).

Também Chapman (2014), ao investigar a relação entre a Sport England e as NGBs, relata que as opiniões das NGBs sobre a Sport England eram mais confusas. Havia preocupações sobre os projetos e prioridades de curto prazo da Sport England, o que significava que as NGBs frequentemente precisam mudar seu trabalho para se alinharem às prioridades da 
Sport England a fim de proteger seu financiamento. Para Berry e Manoli (2018), esse alto nível de dependência dessa fonte única era frequentemente associado a um medo avassalador de um futuro em que esse financiamento fosse retirado, com todas as NGBs subjacentes a esse financiamento central representadas, na época da pesquisa dos autores, por 80-95\% de sua renda total.

Para Berry e Manoli (2018), tensões podem surgir quando existe uma diferença de expectativas entre o organismo financiado e o financiador / governo. Com base no fato de que todos as NGBs entrevistadas argumentaram que suas estratégias foram influenciadas pela estratégia da Sport England, sugerem os autores que o aumento do financiamento do governo pode impactar diretamente a autonomia dos órgãos de governo. Por outro lado, argumentam que esse aumento da dependência, associado a pressões adicionais por mudança, pode ser algo positivo para o esporte em termos de melhorias na governança, conscientização comercial e profissionalismo.

Como as NGBs continuam recebendo mais de $60 \%$ de seu financiamento do governo, podemos ponderar, por meio da Teoria da Dependência de Recursos, para a qual a chave da sobrevivência organizacional é a capacidade de adquirir e manter recursos, que as NGBs, apesar de sua relativa independência, acabam por se sujeitarem às estruturas intermediárias, que repassam tais recursos públicos. Para a escolha do dispositivo menos constrangedor para governar as relações com seus parceiros de troca, o que permitirá maximizar sua autonomia e minimizar a incerteza e a dependência, deveriam as NGBs diversificar suas fontes de recursos e a criação de fóruns de esportes para facilitar a discussão e o compartilhamento de ideias, a fim de alcançar a excelência financeira e operacional (Berry \& Manoli, 2018).

Buscando relacionar a realidade do Reino Unido com a do Brasil, nosso segundo objetivo específico, podemos observar similaridades e diferenças entre os Sistemas Esportivos. Nos dois países estão presentes estruturas que intermediam a relação entre órgãos governamentais e federações esportivas. Enquanto no primeiro, a UK Sport e Sport England cumprem tal função, no Brasil, os Comitês Olímpico e Paralímpico são os responsáveis, como "agentes" representando os "principais", conforme figura abaixo: 
Figura 3 - Sistema Esportivo do Brasil

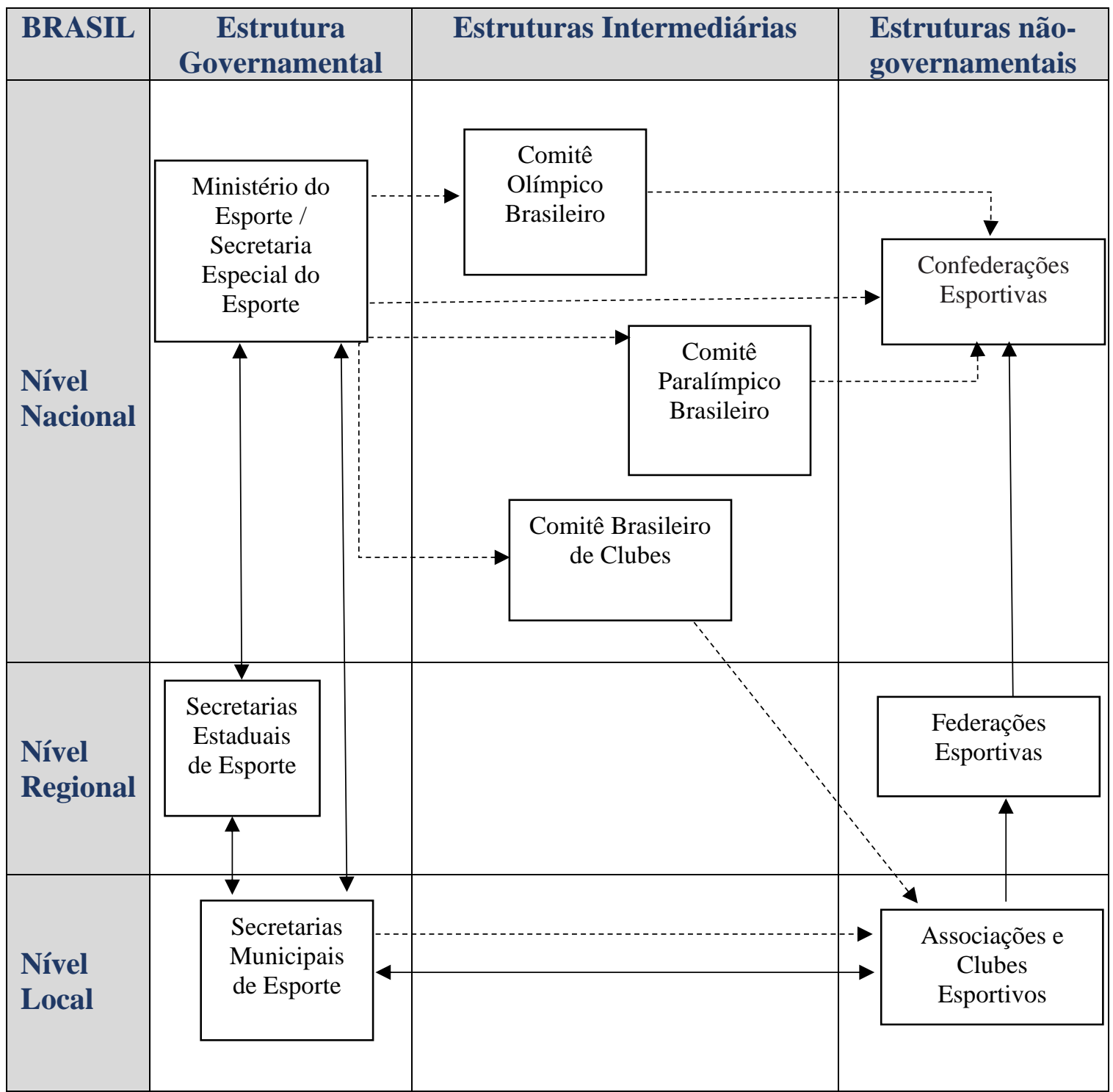

Financiamento

Filiação / Parceria

Relação hierárquica

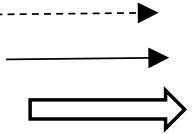

Fonte: Os autores.

A principal diferença é que, no Brasil, pode haver parcerias diretas entre as confederações esportivas e os órgãos governamentais, por meio do patrocínio de empresas estatais, convênios ou da Lei de Incentivo de Esporte (Brasil, 2006). Nesse caso, supre-se a figura do "agente" intermediador, e a relação pode se aproximar mais da perspectiva da cogestão, envolvendo interação direta no setor público e significativa entre o Estado, federações e cidadãos em termos reais nos processos de formulação e implementação de políticas. Todavia, 
essas relações não têm característica permanente, podendo ser adotadas apenas por um curto período de tempo.

Tanto no Brasil como no Reino Unido, as (con)federações esportivas são altamente dependentes do dinheiro público, o que tende a diminuir sua autonomia, considerando a Teoria de Dependência de Recursos.

\section{Conclusões}

O estudo pretendeu apresentar o sistema esportivo do Reino Unido, especificamente na relação entre o Estado e as federações esportivas, buscando explicar sua dinâmica e funcionamento.

Pudemos observar que o Sistema Esportivo do UK é altamente dependente de recursos públicos oriundos da Loteria Nacional e do Tesouro público. É resguardada certa autonomia das entidades esportivas, mas a dependência do dinheiro público leva as instituições a se sujeitarem às diretrizes do governo e das instituições que intermediam a relação entre governo e federações esportivas. Nesse sentido, UK Sports e Sport England assumem um papel central no desenvolvimento esportivo do país. São instituições que concentram recursos financeiros e poder de decisão.

Tal quadro não difere substancialmente do observado no Brasil, onde as confederações esportivas dependem do dinheiro público e têm sua relação com o governo mediada por instituições como o Comitê Olímpico Brasileiro e o Comitê Paralímpico Brasileiro. Há de se considerar que, num contexto de globalização, o pressuposto de que os principais determinantes das políticas públicas estão confinados aos limites dos Estados-nacionais vem perdendo força, enquanto cresce o argumento a favor de que as políticas domésticas vêm sendo cada vez mais influenciadas por redes de políticas supranacionais (Houlihan \& Green, 2008). Segundo Guidens (2003), vivemos em um mundo globalizante, em que informação e imagens são rotineiramente transmitidas através do mundo, estando todos regularmente em contato com outros que vivem e pensam de maneira semelhante ou diferente de nós. Com isso, há de se supor que sistemas esportivos nacionais de diferentes países apresentam semelhanças devido à facilidade de comunicação e troca de informações, que possibilitam processos de policy learning e policy transfer. O uso de recursos de Loterias para financiar o esporte em diferentes países pode ser outro elemento elucidador desse tipo de reflexão. 
Por outro lado, há características no Sistema Esportivo do Reino Unido que diferem do Sistema brasileiro e podem ser alvo de nossa reflexão. O principal deles é a existência, no Sistema do Reino Unido, de uma instituição voltada ao esporte de rendimento ou de elite, a $U K$ Sports, e de outra focada nos aspectos de participação esportiva, a Sport England. Isso mostra, a princípio, um maior equilíbrio, já que, no Brasil, há instituições intermediárias representantes apenas do esporte de elite. Além disso, por meio das diretrizes e financiamento da Sport England, espera-se que as federações esportivas naquele país assumam responsabilidades pelo desenvolvimento esportivo como um todo, desde a participação esportiva, a detecção de talentos e o desenvolvimento do esporte de base, até o esporte de elite. No Brasil, não há determinação semelhante, pois a responsabilidade de promoção e aprimoramento das práticas esportivas de rendimento pelas instituições esportivas privadas (Brasil, 1998) não significa necessariamente o desenvolvimento e a generalização de tais práticas (Canan, Rojo \& Starepravo, 2019).

Enfim, cumpre-se, com isso, o objetivo aqui de apresentar de maneira panorâmica o Sistema Esportivo do Reino Unido e de trazer algumas reflexões iniciais em relação ao contexto brasileiro. A falta de aprofundamento na comparação com o Brasil pode ser considerada uma das limitações do estudo. Abre-se, nesse sentido, uma frente de pesquisa que poderá explorar, com mais profundidade, pontos específicos, como as fontes de financiamento, o nível de autonomia das entidades do terceiro setor, legislação, entre outras, a fim de corroborar ou contrapor os resultados aqui apresentados. Sugerimos que tal empreitada se dê considerando, especificamente, elementos da globalização e da "glocalização" (Giulianotti \& Robertson, 2007), a fim de se pensar as realidades esportivas de cada país e em possíveis comparações.

\section{Referências}

Active Partnership (s. d.). About Us. Disponível em https://www.activepartnerships.org/aboutus, acesso em 21 de dezembro de 2019.

Andersen, S. \& Ronglan, L. (2012). Nordic elite sport: same ambitions - different tracks. Oslo: Universitetsforlaget.

Berry, R. \& Manoli, A. E. (2018) Alternative revenue streams for centrally funded sport governing bodies, International Journal of Sport Policy and Politics, 10:3, 429-450.

Bergsgard, N.A., Houlihan, B., Mangset, P., Nødland, S. I. \& Rommetvedt, H. (2007). Sport policy: A comparative analysis of stability and change. Oxford: Elsevier. 
BOA (s. d.). About us - The British Olympic Association. Disponível em https://www.teamgb.com/about-boa. Acesso em 13 de janeiro de 2020.

Brasil (1998). Lei n. 9.615 de 24 de março de 1998. Institui normas gerais sobre desportos e dá outras providências. Congresso Nacional. Disponível em:

http://www.planalto.gov.br/ccivil_03/leis/L9615consol.htm. Acesso em 20 de dezembro de 2019.

Brasil (2006). Lei $n^{o} 11.438$ de 29 de dezembro de 2006. Dispõe sobre incentivos e benefícios para fomentar as atividades de caráter desportivo e dá outras providências. Congresso Nacional. Disponível em: http://www.planalto.gov.br/ccivil_03/_Ato20042006/2006/Lei/L11438.htm. Acesso em 12 de dezembro de 2019.

Canan, F., Rojo, J., \& Starepravo, F. (2019). Direito ao esporte: possibilidades a partir de políticas multicêntricas, regulatórias e redistributivas. Pensar a Prática, 22.

Chapman, P. (2019). Policy Stability in a Time of Turbulence: The Case of Elite Sport Policy in England/the UK. Tese de doutorado. Loughborough University.

Coghlan, J. F. \& Webb, I. M. (1990) Sport and British Politics Since 1960, Basingstoke: The Falmer Press.

Collins, M. (2008). Public policies on sports development: Can mass and elite sport hold together? In V. Girginov (Ed.), Management of sports development. Oxford: Elsevier, 59 88.

Collins, M. (2010). 'The development of Sport Development' In: Collins M. (ed.). Examining Sports Development, Abingdon: Routledge, 14 - 41.

Couto, A. C., Couto, M., Boschi, C. \& Lemos, K. (2013). Brazil. In: Hallmann, K., and Petry, K., eds. Comparative sport development: Systems, participation and public policy (Sports Economics, Management \& Policy 8). New York, NY: Springer Science, 301-310.

Davis, G. F. \& Cobb, J. (2010). Resource Dependence Theory: Past and future. Research in the Sociology of Organizations, 28 21-42. http://dx.doi.org/10.1108/S0733$\underline{558 X(2010) 0000028006}$

De Bosscher, V. (2016). A mixed methods approach to compare elite sport policies of nations. A critical reflection on the use of composite indicators in the Spliss study. Sport in Society, 21 (2), 331-355.

De Bosscher, V, Shibli, S., Westerbeek, H. \& Van Bottenburg, M. (2015). Successful elite sport policies: an international comparison of the sports policy factors leading to international sporting success (SPLISS 2.0) in 15 nations. Maidenhead: Meyer \& Meyer Sport (UK).

Dowling, M., Brown, F., Legg, D. \& Grix, J. (2018). Deconstructing comparative sport policy analysis: assumptions, challenges, and new directions, International Journal of Sport Policy and Politics, 10:4, 687-704. 
Ebbinghaus, B. (2005). When less is more: selection problems in large-N and small-N crossnational comparisons. International Sociology, 20 (2), 133-152.

Girginov, V. (2017). UK: England - National Governing Bodies of Sport and Government Agencies. In: Scheerder, J., Willem, A. and Claes, E. (2017). Sport policy systems and sport federations: a cross-national perspective / Jeroen Scheerder, Annick Willem, Elien Claes, eds. London, United Kingdom: Palgrave Macmillan.

Giulianotti, R., Itkonen, H., Nevala, A. \& Salmikangas, A.K. (2019). Sport and civil society in the Nordic region. Sport in Society, 22:4, 540-554.

Giulianotti, R. \& Robertson, R. (2007). Sport and globalization: transnational dimensions. Global Networks, 7 (2), pp. 107-112.

Gratton, C., Taylor, P. \& Rowe, N. (2013). UK: England. In: Hallmann, K., and Petry, K., eds. Comparative sport development: Systems, participation and public policy (Sports Economics, Management \& Policy 8). New York, NY: Springer Science, 167-180.

Green, M. (2004). Changing policy priorities for sport in England: the emergence of elite sport development as a key policy concern, Leisure Studies, 23:4, 365-385.

Green, M. (2007). Olympic glory or grassroots development?: Sport policy priorities in Australia, Canada and the United Kingdom, 1960 - 2006. The International Journal of the History of Sport, 24:7, 921-953.

Green, M. \& Houlihan, B. (2005) Elite Sport Development: Policy learning and political priorities, Abingdon: Routledge.

Groeneveld, M. (2009). European Sport Governance, Citizens, and the State. Public Management Review, 11:4, 421-440.

Hallmann, K. \& Petry, K. (eds.), (2013). Comparative sport development: Systems, participation and public policy (Sports Economics, Management \& Policy 8). New York, NY: Springer Science.

Henry, I. (2009). European models of sport: Governance, organisational change and sport policy in the EU. Hitotsubashi Journal of Arts \& Sciences, 50, 41-52.

Houlihan, B. (1991). The Government and Politics of Sport, London: Routledge.

Houlihan, B. (1997). Sport, Policy and Politics: A comparative analysis, London: Routledge.

Houlihan, B. (2000). Sporting excellence, schools and sport development: The politics of crowded policy spaces, European Physical Education Review, 6 (2), 171 - 193.

Houlihan, B. \& Green, M. (eds.) (2008). Comparative Elite Sport Development: systems, Structures and Public Policy, Oxford: Elsevier. 
Houlihan, B. \& White, A. (2002). The Politics of Sports Development: Development of Sport or Development Through Sport? Abingdon: Routledge.

Jeffreys, K. (2012). Sport and Politics in Modern Britain: The Road to 2012, Basingstoke: Palgrave Macmillan.

Keech \& Nauright (2016). The Organisation of Sport in the United Kingdom. In: Zhang, J., Nauright, J. \& Huang, R. (eds.) (2016). Development of the Sport Industry in Leading Economies, Edition: 1: Shanghai University of Sport Press.

Kendall, J. (2010). The Limits and Possibilities of Third Sector Europeanization. Journal of Civil Society, 6:1, 39-65.

King, N. (2009) Sport Policy and Governance: Local Perspectives. Oxford: Butterworth Heinemann.

Lexology (2019). Sports Law in the United Kingdom. Disponível em https://www.lexology.com/library/detail.aspx?g=7cc100e9-382e-4013-b1a0-8499c6889c0e. Acesso em 06 de janeiro de 2020.

Lucidarme, S., Babiak, K. \& Willem, A. (2018). Governmental power in elite sport networks: a resource-dependency perspective. European Sport Management Quarterly, 18:3, 348-372.

Mazzei, L. C., de Barros Meira, T., da Cunha Bastos, F., Silveira Böhme, M. T. \& De Bosscher, V. (2015). High performance sport in Brazil: Structure and policies comparison with the international context. Gestión y Política Pública. Volumen temático 2015, 83-111.

Mezzadri, F. M., Moraes e Silva, M., Figuêroa, K. M. \& Starepravo, F. A. (2015). Sport Policies in Brazil. International Journal of Sport Policy and Politics, 7:4, 655-666.

Nicholson, M., Hoye, R. \& Houlihan, B. (eds.) (2011). Participation in sport: International policy perspectives, Routledge, Abingdon.

Ons (2019). Gross Domestic Product (GDP). Disponível em https://www.ons.gov.uk/economy/grossdomesticproductgdp. Acesso em 07 de janeiro, 2020.

ParalympicsGB (s.d.). How we are funded._Disponível em https://paralympics.org.uk/footerpages/funding. Acesso em 13 de janeiro de 2020.

Parnell, D., May, A., Widdop, P., Cope, E. \& Bailey, R. (2019). Management strategies of nonprofit community sport facilities in an era of austerity. European Sport Management Quarterly, 19:3, 312-330.

Pfeffer, J. \& Salancik, G. R. (1978). The external control of organizations: A resource dependence perspective. New York: Harper \& Row.

Roan, D. \& Nathanson, P. (2018). UK Sport accused of 'bullying' over funding by ex-BBSA directors. Disponível em https://www.bbc.co.uk/sport/winter-sports/46322091. Acesso em 10 de janeiro, 2020. 
Salamon, L. M. \& Flaherty, S. L. Q. (1996). "Nonprofit Law: Ten Issues In Search of Resolution." Working Papers of the Johns Hopkins Comparative Nonprofit Sector Project, no. 20, edited by Lester M. Salamon and Helmut K. Anheier. Baltimore: The Johns Hopkins Institute for Policy Studies.

Scheerder, J., Willem, A. \& Claes, E. (eds.) (2017). Sport policy systems and sport federations: a cross-national perspective / Jeroen Scheerder, Annick Willem, Elien Claes, eds. London, United Kingdom: Palgrave Macmillan.

Shapiro, S. P. (2005). Agency Theory.Annual Review of Sociology, 31:1, 263-284.

Simaens, A. \& Koster, M. (2013). Reporting on sustainable operations by third sector organizations: A signalling approach. Public Management Review,15:7, 1040-1062.

Skille, E. A. (2015). Community and sport in Norway: between state sport policy and local sport clubs. International Journal of Sport Policy and Politics, 7:4, 505-518.

Somerville, P. \& Haines, N. (2008). Prospects for Local Co-Governance. Local Government Studies, 34:1, 61-79.

Sport England (2019a). Results from our latest survey also show inactivity rates decreasing, but stubborn inequalities still remain. Disponível em https://www.sportengland.org/news-andfeatures/news/2019/april/11/record-numbers-of-people-in-england-are-getting-active/. Acesso em 15 de dezembro de 2019.

Sport England (2019b). SPORTS THAT WE RECOGNISE: There is a vast range of activities that people might class as sports. Disponível em https://www.sportengland.org/ourwork/national-governing-bodies/sports-that-we-recognise/. Acesso em 11 de outubro de 2019.

Sport England (2019c). Local Contacts. Disponível em https://www.sportengland.org/aboutus/local-contacts/. Acesso em 17 de outubro de 2019.

Starepravo, F. A., Canan, F. \& Santos, L. L. S. R. (2017). A Panorama on Sports Policies in Brazil. In: Kyros, R. and Lott, M. eds. Public Policy and Social Change: perspectives, challenges and future directions. 1ed.New York: Nova Science Publishers, 63-88.

Stephenson, M. (2013). The third sector and the governance of education. Journal of Educational Administration and History, 45:4, 370-384.

Tacon, R. \& Hanson, A. (2011). Sport policy and the structure of sport in the UK. In: Hassan, D. (org.) (2011). Managing Sport Business: An Introduction. 2 edition. Routledge.

Thing, L. F. \& Ottesen, L. (2010). The autonomy of sports: negotiating boundaries between sports governance and government policy in the Danish welfare state. International Journal of Sport Policy and Politics, 2:2, 223-235.

UK (1995). Sport: Raising the Game. London: Department of National Hertitage.

UK (2015). Sporting Future: A New Strategy for an Active Nation. London: Cabinet Office. 
UK Sports (2019). Faqs. Disponível em https://www.uksport.gov.uk/about-us/faqs. Acesso em 17 de outubro de 2019.

Vos, S., Wicker, P., Breuer, C. \& Scheerder, J. (2013). Sports policy systems in regulated Rhineland welfare states: similarities and differences in financial structures of sports clubs. International Journal of Sport Policy and Politics, 5:1, 55-71.

Walker, C. M \& Hayton, J. W. (2018). An analysis of third sector sport organisations in an era of 'super-austerity'. International Journal of Sport Policy and Politics, 10:1, 43-61.

Walters, G., Tacon, R. \& Trenberth, L. (2011) The Role of the Board in UK National Governing Bodies of Sport, London: Birkbeck Sport Business Centre

World Bank (2019). Urban Population. Disponível em https://data.worldbank.org/indicator/SP.URB.TOTL.IN.ZS. Acesso em 17 de outubro de 2019. 